\title{
Test Method for Command and Control Modes for the Attack of Time-Sensitive Targets Based on Agent
}

\author{
Zhuyun Duanmu \\ Science and technology on information systems \\ engineering laboratory \\ Nanjing, China \\ E-mail: fairydm@qq.com
}

\author{
Jianyu Song \\ Nanjing research institute of electronics engineering \\ Nanjing, China \\ E-mail: minlet2011@gmail.com
}

\begin{abstract}
For the test problem of command and control mode for the attack of time-sensitive targets, this paper proposed a test method for command and control modes for the attack of time-sensitive targets based on Agent, then in the attack of time-sensitive targets as the background, established decider, sensor and shooter of three agents, and combined with three different command and control model, established the operation rules of different Agents. On the basis, constructed simulation test platform, carried out multiple sets of tests, and analyzed the test data.
\end{abstract}

Keywords- time-sensitive targets; command and control mode; simulation test platform; Agent

\section{INTRODUCTION}

The US department of defense defines time sensitive targets (TST) as: an immediate attack goal due to dangerous need, military interpreted as in a relatively short period of time with high military value goal [1]. Complete process of the attack of time-sensitive targets including finding, identifying and tracking, decision making, belligerent, and evaluation of six stages. Compression of the above six stages of attack chain, that is, destroy time-sensitive targets in the shortest possible "attack window" or before the target come into play, can implemented by reducing the time of the each link and reducing the link between transition time to achieve.

The attack system of time-sensitive targets includes sensor platform, decider platform and shooter platform, command and control modes for the attack of time-sensitive targets by changing the interaction between the platform and the decision-making authority between cutting platform in order to compress the attack chain, ensuring destroyed timesensitive targets within the time window. Command and control modes for the attack of time-sensitive targets include decider-led mode, sensor-led mode and shooter-led mode. U.S. military attaches great importance to the study of the use of mathematical analysis methods and simulation methods to assess operational effectiveness issues sensitive target strike missions. Rand Company achieved a lot of research results in this area, such as the "network-centric warfare performance metrics" as the representative of the many reports presented multiple evaluation index of the missions of the attack of time-sensitive targets, and announced some selective testing and analysis results. However, for technical reasons of confidentiality, these reports lack of assess the specific implementation steps of the detailed description.

For the test problem of command and control modes for the attack of time-sensitive targets, this paper proposes an agent-based test method of command and control modes for the attack of time-sensitive targets which simulation modeled Command and control modes for the attack of time-sensitive targets, describes the corresponding operate rules of decider Agent, sensor Agent and shooter Agent running allegations mode respectively. On this basis, builds a simulation platform for multi-group test, and analyzes the test data.

\section{COMMAND AND CONTROL MODES FOR THE ATTACK OF TIME-SENSITIVE TARGETS}

The strategies of the attack of time-sensitive targets include tactical grouping and command and control mode in which the tactical group including the weapons platforms of attack targets, carrying ammunition, sensor and tactical action. Command and control mode include the decider-led mode, sensor-led mode and shooter-led mode. Each command and control model has different requirements of performance of weapons; ammunition and sensor, which is a constraint of choose combat platforms to generation combat countermeasures set. In addition to command and control model is the platform of choice for fighting outside the constraints, but also the platform for decision-making and operational information access has been limited, optimize operational processes to ensure destroy sensitive targets within the time window [2].

(1)The traditional command and control model is the decider-led mode, command center controls the decider and the flow of information, intelligence and plans were submitted to the layers, the layers of decisions issued by the highest decider. Sensor and shooter have no decision-making power. Its advantage is through real-time sensor's command, in a timely manner to determine the target feature, the information is sent to the shooter to combat timely. The disadvantage is that decider control the flow of information. Mainly applicable to the fixed attack of time-sensitive targets which information from the national level.

(2) In the command and control mode of sensor-led, the sensor platform has a certain decision-making powers, issued instructions to shooter platform. The advantage is that the sensor platform directly through real-time situational awareness, improve the speed of command and control and shorten the decision-making cycle. Further, since the 
recognition and decision-making process is done on the sensor platform, the platform is greatly reduced conversion time variable. The disadvantage is that the high performance requirements of the sensor platform. Mainly applicable to improve the Ground Controlled Interception technology.

(3) In the command and control mode of shooter-led, the shooter platform execute attack tasks based instruction decider platform is authorized. These instructions authorization shooter platform with a certain decisionmaking powers may be required to respond to the sensor platform. Its advantage is attack fastest. Since the decisionmaking and engagement both on the shooter platform, which greatly reduces the conversion time variable platform. The disadvantage is that the shooter platform for high performance requirements. Mainly applicable to discover the target of to need is to destroy.

\section{AGENT-BASED TEST PLATFORM ARCHITECTURE}

Multi-Agent-oriented modeling and simulation technology is one of the most dynamic methods and influence. The attack of time-sensitive targets command and control test platform based Agent uses agent federal structure, as shown below, including intelligence simulator, Agent registry center, resource scheduling / communication centers, data acquisition module and a plurality of sensor Agent, decider Agent, weapon platform Agent and TST Agent. This structure helps to expand and maintain the system, so that a clear structure of the program, with high efficiency.

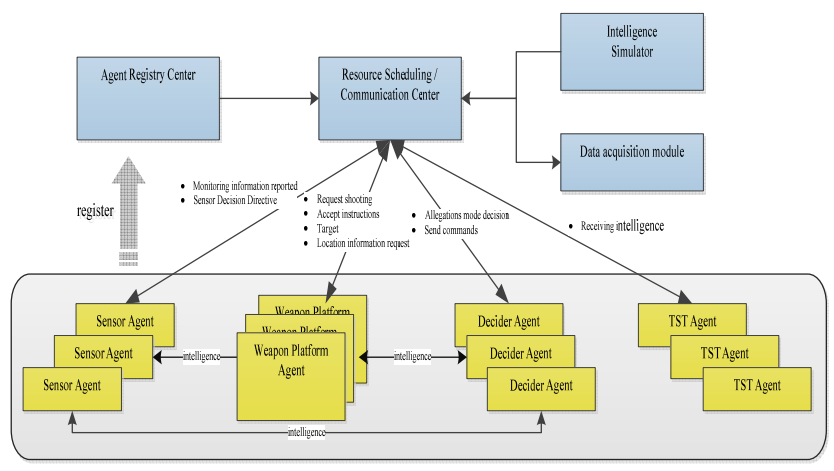

Figure 1. Method Schematics of the test platform of command and control modes for the attack of TST

Agent Registry Center provides registry, query and modify of all kinds Agent information in the attack of timesensitive targets. Resource Scheduling / communications agent is an abstraction of the upper Agent, which is not responsible for any object in simulated battlefield, but is responsible for creating and destroying objects Agent system, and is responsible for communication and messaging between different Agent distribution, while it also bear the send key messages to the data acquisition module, logging and other functions.

Decider Agent is command and control center in the attack of time-sensitive targets, according to the current battlefield combat situation, the use of grouping rules and the corresponding countermeasures to combat reasoning mechanism to select the appropriate combat platforms from the Agent registry to generate battle plans, and choose the best combat allegations mode. The main goal Agent makers responsible for receiving information, all kinds of attacks request command, damage assessment information, and send all kinds of attack command in the decision-makers mode.

Sensor Agent, shooter Agent correspond the sensor platforms and weapons platforms in the attack of timesensitive targets. Sensor Agent is responsible for tracking the battlefield to detect time-sensitive targets, depending on intelligence allegations mode will be sent to a different Agent; shooter Agent is responsible for management and control of one's own firepower, and make requests submitted shooting, targets attacking and other acts. The TST Agent receive intelligence simulator to maintain and update the battlefield sensitive target information.

\section{THE PRINCIPLE OF EMULATION TEST METHODS}

Then focus on the common Agent model, the operating rules of the different roles Agent on the test platform.

\section{A. Test Platform Agent Model}

Test platform Agent model shown below, including behavior, attributes, inference mechanism and rule base module. Behavior module includes perception, decisionmaking, assessment, communication, flight and combat such action; attributes include static attributes and dynamic attributes, static attributes include the role setting, decider model attributes, sensor model attributes and shooter model attributes, and dynamic attributes include flight attributes, equipment attributes, and the current task information. The core modules of Agent model are inference mechanism and rule base, inference mechanisms include the judgment of attack countermeasures, attack link optimization and cost efficiency comparison, etc.; Agent rule base includes countermeasures to combat the rule change, decider-led mode rules, sensor-led mode rules and shooter-led mode rules.

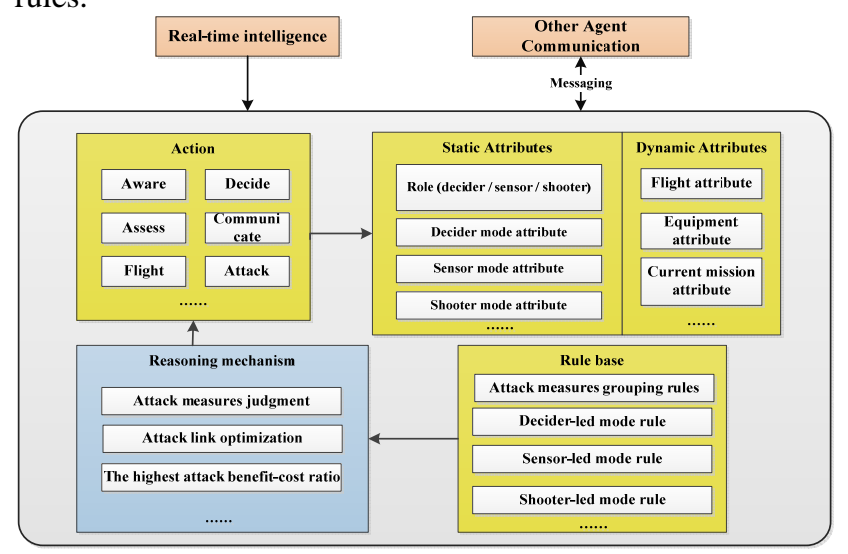

Figure 2. Test platform Agent model

Agent starts to enter the ready state after boot, based on real-time intelligence received, own attribute, and other 
information sent by Agent, in accordance with the rule base and inference mechanism to determine their own behavior; addition, the occurrence of Agent may adjust the dynamic behavior of the Agent's attribute values, as well as communicate with other Agent occurred.

\section{B. Test Platform Agent operating rules}

In the multi-Agent system, depending on the different role, the interactive rules and way between the various Agents are also very different. The test platform to the attack of time-sensitive targets as the background, establish the decider, sensor and shooter of three roles Agent, and different operating rules of Agents combined with three different command and control modes. As shown below, followed by operating rules of decider Agent, sensor Agent and shooter Agent.

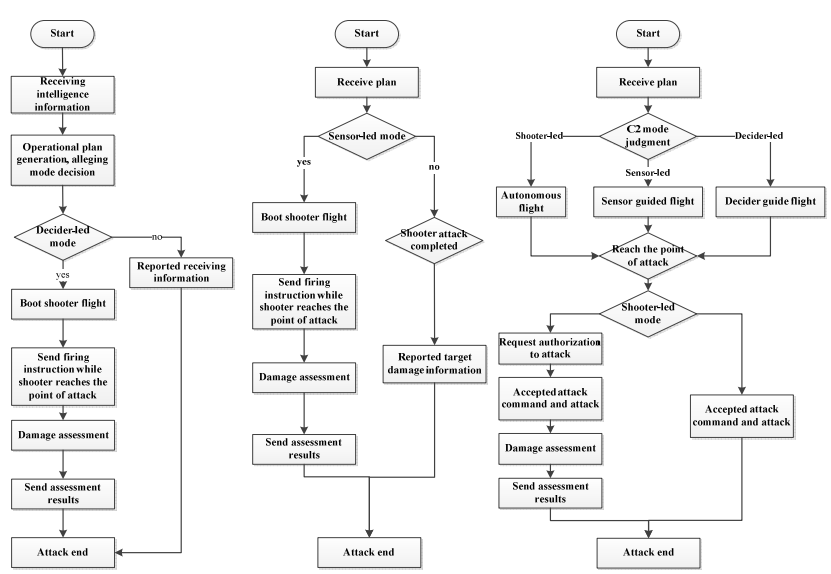

Figure 3. Different roles Agent operating rules

(1) Decider Agent receives intelligence information, the use of countermeasures to combat grouping rules and reasoning mechanism to generate the corresponding operational plans, choose the best mode to combat allegations. Under the decider-led mode, decider Agent guide shooter flight, send fire command reaching the point of attack, and returned conduct damage assessment information, and send the results of the assessment to inform each Agent the end of war. Under the non-decider-led mode, deciders Agent reported receiving intelligence information only, and make the appropriate response.

(2)After receiving the plan from decider Agent, under the sensor-led mode, the sensor Agent guide shooter flight, send fire command reaching the point of attack, and returned conduct damage assessment information, and send the results of the assessment to inform each Agent the end of war. Under the non-sensor-led mode, the sensor report the detect target information to decider Agent.

(3) Shooter Agent flight to the point of attack under shooter-led mode autonomously, flight to the point of attack guided by decider under the decider-led mode. After reaching the point of attack, the shooter Agent under shooterled mode request for authorization from decider agent, then attack while receiving attack command, assess real-time damage; Under the non-shooter-led mode, shooter Agent receives attack command and attack.

\section{TEST DATA ANALYSIS}

On the test platform, to be set the TST's type is missile launchers and ground mobile command vehicles, etc., and set the target of weapons loads, elements of movement and status information such as the time window, loaded onto the test platform for multiple tests . By finishing the analysis of test data can be found, in three different modes allegations, shooter mode execution time is almost always the shortest, followed by the sensor mode, decider mode is longest, which is different from the theoretical analysis conclusion allegations coincide.

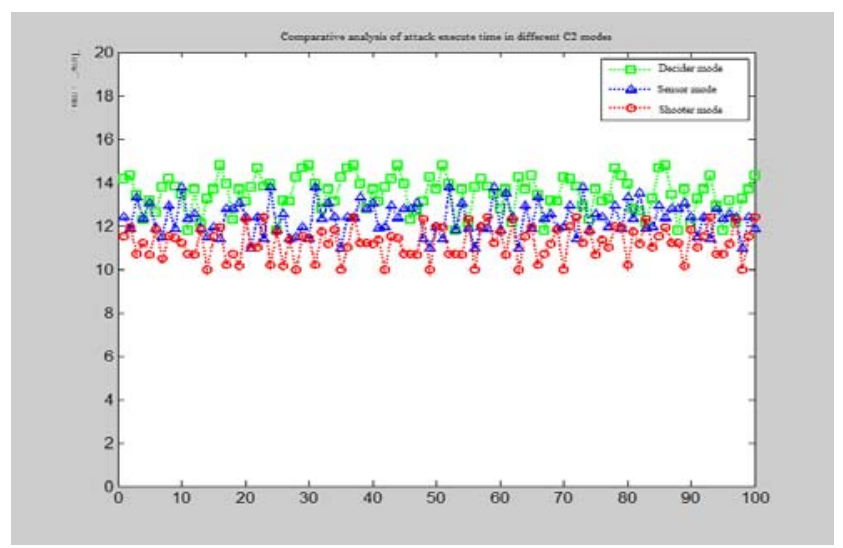

Figure 4. Comparative analysis of attack execute time in different C2 modes

\section{CONCLUSION}

The attack of time-sensitive targets is a very difficult and complex operational task, to complete the combat mission quickly and efficiently, it must be rational allocation of resources to the war, and enhance information exchange and collaboration tasks between the various platform resources. This paper established the appropriate role Agent mode for the various platform resources, designed the test platform architecture based on Agent, carried out and analyzed in the ideal case multiple tests. Due to the complexity of the attack of time-sensitive targets, certain of difficulty for the system performance analysis and simulation methods based Agent is brought. Agent technology can reflect systemic combat system elements, but because of the large number of equipment and complex relationships in the attack of timesensitive targets system, the difficulty of Agent simulation increased. Therefore, the establishment of a relatively complete system of the attack of time-sensitive targets Agent mode base is the next focus of the work, on this basis, should continue to improve the inference mechanism and rule base of the test platform Agent mode in order to improve the logic of Agent simulation model. 


\section{REFERENCES}

[1] Hebert A. J. Compressing the kill chain[J] Air Force Magazine, March 2003.

[2] LI Min-yong,SUN Sheng-chun.Operational Mechanism Analysis for Hunting Time-Sensitive Targets[J].Information Command Control System \& Simulation Technology,2004,26(2):10-15.(in Chinese)

[3] ZHANG Kai-de, ZHAO Shu-bin.The Command and Control Technology of Time Critical Strike [J]. Command Control \& Simulation, 2006, 4 (2):1-5. (in Chinese)
[4] CHEN Jun, GAO Xiao-guang, DING Lin. Operational Effectiveness Evaluation and Cost-effectiveness Analysis of System for Destroying Time-sensitive Target [J]. Journal of System Simulation,2007,19(17):4042-4044. (in Chinese)

[5] Jia Xinqiang,Sui Jiangbo,Gao Bo. Agent Model of Hardkill and Softkill Weapon Resourc[J]. Ship Electronic Engineerin, 2011, 31(4) : 105-108. (in Chinese)

[6] JIN Hong, ZHOU Wu , LI Bei-lin .Priority Attacking Sequence of Time-sensitive Targets [J]. Command Control \& Simulatio,2009, 31(5):90-97. (in Chinese). 